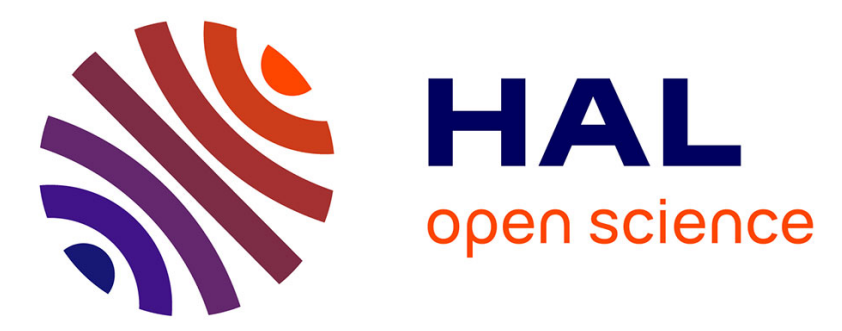

\title{
Subharmonic steps in the I-V characteristic of short microbridges due to non-sinusoidal current-phase relations
}

\author{
H. Lübbig, H. Luther
}

\section{- To cite this version:}

H. Lübbig, H. Luther. Subharmonic steps in the I-V characteristic of short microbridges due to non-sinusoidal current-phase relations. Revue de Physique Appliquée, 1974, 9 (1), pp.29-34. 10.1051/rphysap:019740090102900 . jpa-00243762

HAL Id: jpa-00243762

https://hal.science/jpa-00243762

Submitted on 1 Jan 1974

HAL is a multi-disciplinary open access archive for the deposit and dissemination of scientific research documents, whether they are published or not. The documents may come from teaching and research institutions in France or abroad, or from public or private research centers.
L'archive ouverte pluridisciplinaire HAL, est destinée au dépôt et à la diffusion de documents scientifiques de niveau recherche, publiés ou non, émanant des établissements d'enseignement et de recherche français ou étrangers, des laboratoires publics ou privés. 


\title{
SUBHARMONIC STEPS IN THE I-V CHARACTERISTIC OF SHORT MICROBRIDGES DUE TO NON-SINUSOIDAL CURRENT-PHASE RELATIONS
}

\author{
H. LÜBBIG and H. LUTHER \\ Physikalisch-Technische Bundesanstalt, Braunschweig und Berlin, Germany
}

\begin{abstract}
Résumé. - Nous étudions l'influence des harmoniques de rang élevé $\left(I_{k} \sin k \varphi, k>1\right)$ de la relation courant-phase $I(\varphi)$ d'un micropont supraconducteur sur la caractéristique $I-V$ obtenue en haute fréquence. Nous calculons à l'aide d'une relation non sinusoïdale $I(\varphi)$ dérivée du modèle de Baratoff et al., la hauteur des marches en fonction de l'amplitude du champ de haute fréquence. Nous discutons une méthode d'approximation pour déterminer le spectre $I_{k}$ de $I(\varphi)$ à partir d'une caractéristique structurée en marches préalablement donnée.
\end{abstract}

Abstract. - The influence of high order harmonics $\left(I_{k} \sin k \varphi, k>1\right)$ of the current-phase relation $I(\varphi)$ of a short superconducting microbridge on the corresponding rf-induced currentvoltage characteristic is studied. For a special non-sinusoidal relation $I(\varphi)$ derived from the model of Baratoff et al., the step-heights are calculated as function of the amplitude of the applied rf-field. An approximation method for the determination of the spectrum $I_{k}$ of $I(\varphi)$ by means of an evaluation of the given step-structure is discussed.

1. Introduction. - Due to the periodicity of the Josephson-relationship between the pair-current $I$ and the phase-difference $\varphi$ of the order parameters of two weakly coupled bulk superconductors, constantvoltage steps in the corresponding current-voltage characteristics may arise if a rf-field is applied to the junction. Some of the steps occur at mean voltages $\bar{V}_{m}=m\left(h f_{\text {rf }} / 2\right.$ e $)$ as a result of phase-locking between the applied radiation $\left(f_{\mathrm{rf}}\right)$ and the fundamental selfoscillation component of the system of the frequency $f_{0}=(2 \mathrm{e} / h) \bar{V}(\mathrm{e}$ denotes the electron-charge, $h=2 \pi \hbar$ Planck's constant, $m$ an integer). These steps are known as principal steps. If furthermore high order harmonics of the frequencies $n f_{0}(n>1)$ are generated, they may be phase-locked by the applied radiation producing additional steps at mean voltages

$$
\bar{V}_{m / n}=\frac{m}{n} \frac{h f_{\mathrm{rf}}}{2 \mathrm{e}}, \quad(n>m),
$$

the so-called subharmonic steps.

For different types of weakly coupling junctions, rf-induced current-voltage characteristics have been calculated and partly compared with experimental results [1] to [4]. For the special case of superconducting microbridges, previous calculations of step-structured current-voltage characteristics [1], [2], are based on the assumption of a sinusoidal relationship

$$
I(\varphi)=I_{1} \sin \varphi \text {. }
$$

A theoretical analysis of the phase-coupling in microbridges carried out by Baratoff, Blackburn and Schwartz [5], Bardeen and Johnson [6], and Rieger,
Scalapino and Mercereau [7] indicate that a currentphase relation $I(\varphi)=\sum_{k} I_{k} \sin k \varphi$ can be expected in general including high order harmonic components $I_{k} \sin k \varphi(k>1)$, with respect to the phase-difference $\varphi$.

The influence of different non-sinusoidal $I(\varphi)$ relations on the current-voltage characteristics of a junction driven by a dc-current has been studied recently by Auracher and van Duzer [8].

Here we report on results of calculations concerning the influence of a special non-sinusoidal $I(\varphi)$-relation on the step-structure of the rf-induced current-voltage characteristics of short microbridges.

It is the main purpose of our investigations to study the generation of subharmonic steps due to the additional components $I_{k} \sin k \varphi(k>1)$, and to look for a relationship between the step-heights and the amplitudes $I_{k}$.

For the calculations, we have used a $I(\varphi)$-relation given in the article of Baratoff, Blackburn and Schwartz [5]. The model proposed by them is based on the conception that the materials of the coupling zone (B) and the bulk regions (A) of the device are distinguishable only by the mean free paths $l_{\mathbf{B}}<l_{\mathrm{A}}$ and, consequentely, by the (Ginzburg-Landau) coherence-lengths $\xi_{\mathrm{B}}<\xi_{\mathrm{A}}$. A one-dimensional treatment of the corresponding Ginzburg-Landau equations shows that a superconducting current $I$ passing through such a contact causes a marked depression of the order parameter in the interior of the coupling zone accompanied by the formation of a phase-difference $\varphi$ across the junction. The current-phase relation $I(\varphi)$ is determined completely by the weakness-para- 
meter $\gamma=\left(\xi_{\mathrm{B}} / \xi_{\mathrm{A}}\right)^{1 / 2}$ and the normalized bridge length $d=L / 2 \xi_{\mathrm{A}}$. Since the weakness-parameter $\gamma$ is sensitive to variations of the mean free paths, the $I(\varphi)$-relation can be changed by alteration of the impurity of the bridge material. If the condition $\gamma<d<\gamma^{1 / 2}$ is satisfied, a sinusoidal current-phase relation can be expected [9]. For increasing coupling strength, this means that for larger values of the weakness-parameter $\gamma$ (with respect to a fixed effective bridge-length $d$ ), the sinusoidal current-phase relation is modified by additional components

$$
I_{k} \sin k \varphi(k>1) .
$$

In order to study the influence of such modifications on the rf-induced current-voltage characteristics we have chosen as an example the $I(\varphi)$-relation plotted in figure 1 with $\gamma=0.10$ and $d=0.05$. In the lower



Fig. 1. - Current-phase difference relationship $I(\varphi)$ for a short microbridge with $d=0.05, \gamma=0.10$ given in reference [5] and the corresponding normalized amplitudes $I_{k}$.

part of figure 1, the corresponding amplitudes $I_{k}$ of the spectrum of $I(\varphi)$ are shown. These amplitudes may be regarded as a measure of the weakness of the coupling of the microbridge.

If one assumes the quasiparticle current to be governed by añohmic relation, the following currentbalance equation

$\frac{\hbar}{2 \mathrm{e} R_{\mathrm{q}}} \frac{\mathrm{d} \varphi}{\mathrm{d} t}+\sum_{k} I_{k} \sin k \varphi=I_{\mathrm{dc}}+\hat{I} \cos \left(2 \pi f_{\mathrm{rf}} t\right)$

determines the voltage

$$
V(t)=\frac{\hbar}{2 \mathrm{e}} \frac{\mathrm{d} \varphi}{\mathrm{d} t}
$$

across the irradiated junction. In eq. (1) $R_{\mathrm{q}}$ denotes the resistance for the quasiparticle current. The effect of the external field on the junction is described by the monochromatic current on the right hand side of eq. (1) assuming a current source of very high impedance [1], [4].

2. Calculations. - The current-balance eq. (1) has been studied with the aid of an analog-computer. Current-voltage characteristics were calculated for various values of the quasiparticle resistance $R_{\mathrm{g}}$ $\left(2.5 \mathrm{~m} \Omega \leqslant R_{\mathrm{q}} \leqslant 20 \mathrm{~m} \Omega\right)$, and the rf-amplitude $\widehat{I}$ $(0 \leqslant \hat{I} \leqslant 20 \mathrm{~mA})$. The frequency of the rf-field was chosen to be $f_{\mathrm{rf}}=1 \times 10^{10} \mathrm{~s}^{-1}$. A special effort was required for the treatment of the continuously increasing function $\varphi(t)$ in connection with the generation of the non-sinusoidal periodical current-phase relation $I(\varphi)$, figure 1 . This problem was solved by using two integrators for the operation $\varphi=\int \dot{\varphi} \mathrm{d} t$ which were switched alternatively between the computing and the reset state. Further details of this circuit are given in reference [10].

3. Results. - A selection of current-voltage characteristics plotted in figure 2 shows the dependence of the step-structure on the quasiparticle resistance in the range $2.5 \mathrm{~m} \Omega \leqslant R_{\mathrm{q}} \leqslant 20 \mathrm{~m} \Omega$ for a fixed value of $\mathrm{rf}$-amplitude $\hat{I}=6 \mathrm{~mA}$. Applying the relation between the maximum zero-voltage current and the quasiparticle resistance given in reference [3], eq. (2), the corresponding variation of the temperature for a Sn-microbridge can be estimated to cover a range of about $0.1 \mathrm{~K}$ just below the transition temperature. Principal steps at voltages $\bar{V}_{m}=m\left(h f_{\mathrm{rf}} / 2 \mathrm{e}\right)(m=1,2,3)$, and subharmonic steps between them are visible in figure 2. The dependence of the height of the

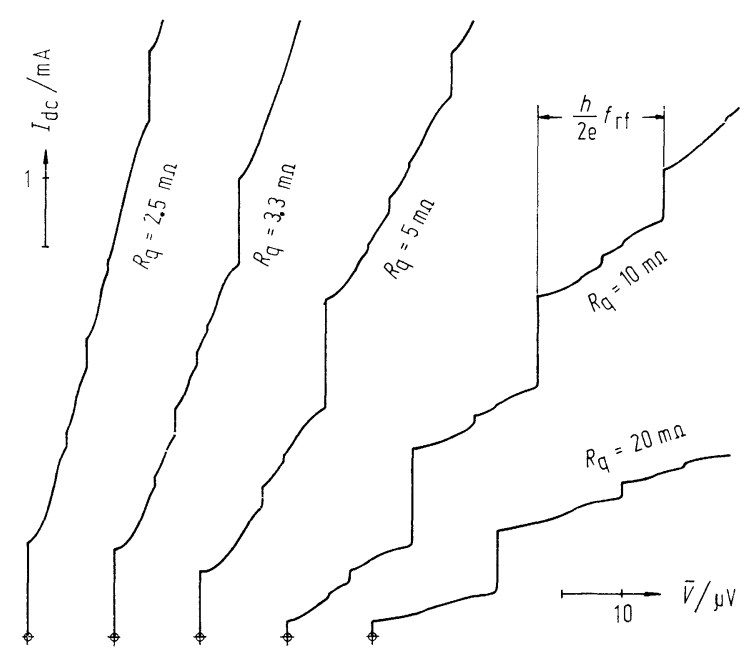

FIG. 2. - Calculated step-structured current-voltage characteristics due to $I(\varphi)$ of figure 1 for different values of the quasiparticle resistance $R_{\mathrm{q}}$ and $\hat{I}=6 \mathrm{~mA}, f_{\mathrm{rf}}=1 \times 10^{10} \mathrm{~s}^{-1}$. The origins are displaced along the $\bar{V}$-axis. 
subharmonic steps on $R_{\mathrm{q}}$ is similar to the behaviour of the principal ones. For a fixed rf-amplitude $\hat{I}$ the height of each step reaches a maximum value for a corresponding special value of $R_{\mathrm{q}}$.

For the lowest value $R_{\mathrm{q}}=2.5 \mathrm{~m} \Omega$, the dependence of the step-heights on the rf-amplitude $\hat{I}$ was studied. In figure 3 the zero-voltage current and the height of the first principal step $(m=1)$ and several of the subharmonic steps $(n=2, \ldots, 5)$ in between them

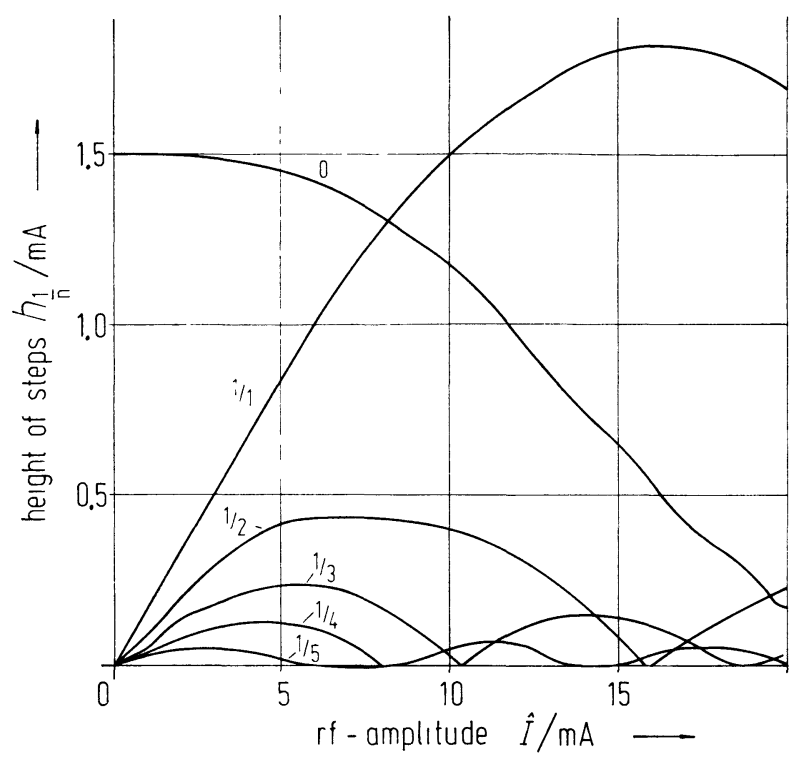

FIG. 3. - The zero-voltage current (0) and the first principal step $(n=1)$ and several subharmonic steps $(1<n \leqslant 5)$ as

functions of $\hat{I}$ due to $I(\varphi)$, figure 1 , and $R_{\mathrm{q}}=2.5 \mathrm{~m} \Omega$.

are shown as function of $\hat{I}$. All step-heights are oscillating functions of $\hat{I}$ as is known from the commonly studied case $I(\varphi)=I_{1} \sin \varphi$. As conspicuous features which differ from the normal case, one observes that the zero-voltage current (0) cannot be suppressed completely in this range of $\widehat{I}$ and that the maximum height of the first principal step (1/1) exceeds that of the zero-voltage current. In addition, it can be seen that the minimum values of the subharmonic stepheights $(1 / n, n>1)$ are displaced towards smaller values of $\hat{I}$ for larger values the subscript $n$.

4. Discussion. - The main features of the behaviour of the step-height as a function of $\widehat{I}$, figure 3 , can be explained qualitatively by the influence of the amplitudes of the periodic voltage $V(t)$ at the step on the mean value of the pair-current $\bar{I}(\varphi)$. In the present case of a non-sinusoidal $I(\varphi)$-relation, figure 1 , the analog-computations indicate that the rf-voltage generated at the zero-voltage current $(\bar{V}=0)$ and at the first principal step $\left(\bar{V}=h f_{\mathrm{rf}} / 2 \mathrm{e}\right)$ is determined mainly by the component $v_{1} \cos \left(2 \pi f_{\mathrm{rf}} t-\theta_{1}\right)$, whereas at the subharmonic steps

$$
\left(\bar{V}=(1 / n)\left(h f_{\mathrm{rf}} / 2 \mathrm{e}\right), n>1\right),
$$

this component is superimposed by the subharmonic term $v_{1 / n} \cos \left(2 \pi f_{\text {rf }} t / n-\theta_{1 / n}\right)$. Then, the voltage $V(t)$ as a solution of eq. (1) depends on four parameters $p_{j}$, i. e. the amplitudes $v_{1}, v_{1 / n}$ and the corresponding phase-angles, $\theta_{1}, \theta_{1 / n}$, which are continuous functions of the parameters $R_{\mathrm{q}}, I_{k}(k=1,2, \ldots)$ and $I_{\mathrm{dc}}$, $\widehat{I}$ in the current-balance eq. (1). If the dc-current is running along one constant-voltage step, the parameters $p_{j}$ vary as functions of $I_{\mathrm{dc}}$ for fixed values of the junction-parameters $R_{\mathrm{q}}, I_{k}$ and the rf-amplitude $\hat{I}$. Accordingly, the height

$$
h_{1 / n}=\bar{I}_{\max }(\varphi)-\bar{I}_{\min }(\varphi)=\sum_{k} I_{k} \Delta \overline{\sin k \varphi}
$$

of the step is determined by the values of the $p_{j}$ related to the extreme values of $I_{\mathrm{dc}}$ which belong to the fixed average voltage $\bar{V}$. In order to facilitate further analysis, one may assume that the variation of the voltage along the step occurs mainly through changes of the phase-angles whereas the amplitudes remain nearly constant. This somewhat oversimplified model is useful, however, to explain main features of the $h_{1 / n}(\widehat{I})$-dependence.

On all these conditions, the contributions of the different amplitudes $I_{k}(k=1,2, \ldots)$, of the $I(\varphi)$ spectrum to the mean value $\bar{I}(\varphi)$ of the pair-current are weighted by the coefficients

$$
\overline{\sin k \varphi}=J \mathrm{~m}\left\{\begin{array}{c}
J_{0}\left(k \zeta_{1}\right) \exp i k\left(\varphi(0)+\zeta_{1} \sin \theta_{1}\right) \\
\text { for } \bar{V}=0 \\
(-1)^{k} J_{k}\left(k \zeta_{1}\right) \times \\
\times \exp i k\left(\varphi(0)+\theta_{1}+\zeta_{1} \sin \theta_{1}\right) \\
\text { for } \bar{V}=\frac{h f_{\mathrm{rf}}}{2 \mathrm{e}}, \\
\zeta_{1}=\left(2 \mathrm{e} v_{1} / h f_{\mathrm{rf}}\right),
\end{array}\right.
$$

at zero-voltage and at the voltage of the first principal step respectively. The $J_{\mathbf{j}}$ denote ordinary Besselfunctions of integer order.

These equations show that the zero-voltage current has its largest maximum at $\zeta_{1}=0$ whereas the first principal step (and all higher ones) vanishes in this point, trivially. Since the set of junction and rf radiation parameters which were used in the analogcomputer calculations of eq. (1) results in a maximum zero-voltage current which equals $I_{1}$, as shown in figure 3 , it is not surprising that the maximum height of the first principal step

$$
h_{1, \max } \lesssim\left(\sum I_{k}^{2}\right)^{1 / 2}
$$

exceeds this value because of the contributions of the high order amplitudes $I_{k}(k>1)$. Furthermore, it can be deduced from eq. (3) that a vanishing zero- 
voltage current requires the $J_{0}\left(k \zeta_{1}\right)$ to be zero for all integers $k \geqslant 1$. This condition, however, cannot be satisfied for a single value of $\zeta_{1}$ since the zeros $z_{s}(s=1,2, \ldots)$, of $J_{0}(z)$ are distributed inhomogenuously. Therefore, the zero-voltage current (and the principal steps too) can be expected to be finite for finite rf-amplitudes $\widehat{I}$.

For subharmonic steps, the weight-factors are given by

$$
\begin{aligned}
\overline{\sin k \varphi}= & \sum_{r=-\infty}^{\infty}(-1)^{k+n r} J_{r}\left(k \zeta_{1}\right) J_{k+n r}\left(k n \zeta_{1 / n}\right) \sin \alpha_{r} \\
\text { for } \bar{V}=\frac{1}{n} \frac{h f_{\mathrm{rf}}}{2 \mathrm{e}}, \quad \text { (5) } & \\
\alpha_{r}=(k+n r) \theta_{1 / n}-r \theta_{n}+ & +k\left(\varphi(0)+\zeta_{1} \sin \theta_{1}+n \zeta_{1 / n} \sin \theta_{1 / n}\right), \\
\zeta_{1 / n}= & \left(2 \mathrm{e} v_{1 / n} / h f_{\mathrm{rf}}\right), \quad n>1 .
\end{aligned}
$$

The Bessel-coefficients $J_{k+n r}\left(k n \zeta_{1 / n}\right)$ show that the oscillations of the heights $h_{1 / n}$, in figure 3, of subharmonic steps become more frequent with ascending integer $n$ and, correspondingly, their extreme values are displaced towards smaller rf-amplitudes.

Furthermore, the maximum values $h_{1 / n \text {, max }}$ of the step-height $h_{1 / n}$ with respect to $\widehat{I}$ are approximately linear functions of the spectral components $I_{k}$, as can be shown as follows :

Applying the simplifying model the values $p_{j, \max }$ determining the extreme value of the mean paircurrent are given by $\partial / \partial p_{j}(\overline{\sin k \varphi})=0,(j=1,2)$, if the $p_{j}$ are assumed to be independent from each other. Of course, this is not true exactly since there exists a weak interdependence by means of the currentbalance equation. However, if in addition one takes into account the fact that the mean pair-current varies slowly in the region near its extreme values with respect to $p_{j}(j=1,2)$, one may accept the condition concerning the $p_{j, \max }$ as a good approximation. Consequently, the weight-factors (3 to 5) corresponding to $p_{j, \max }$ are nearly independent of the currentamplitudes $I_{k}(k=1,2, \ldots)$, and, therefore, the mean current related to them is a linear functions of the $I_{k}$ approximately.

In figure 4 , the linear dependence of $h_{1 / 2}\left(I_{2}\right)$ is shown as a result of an analog-computation where the other spectral components and $R_{\mathrm{q}}$ were chosen as before. For $I_{2}=0$ the contribution of the other harmonic components $I_{k} \overline{\sin k \varphi}(k \neq 2)$ is visible, mainly the influence of $k=4$.

Based upon the linearity of the relationship $h_{1 / n, \max }\left(I_{n}\right)$, a linear-interpolation scheme can be used to estimate the spectral components $I_{k}(k=1,2, \ldots)$, of the $I(\varphi)$-relation of the given maximum step-heights $h_{1 / n, \max }$ : as a rough first order approximation one may use a set of $I_{k}^{(0)}$ which equal the given maximum step-heights $h_{1 / n, \max }$ directly. The current-voltage characteristics associated with $I_{k}^{(0)}(k=1,2, \ldots)$, can be calculated on the basis of the current-balance equa-



FIG. 4. - The maximum height $h_{1 / n, \max }$ of the second subharmonic step as function of the amplitude $I_{2}$ of the second harmonic component $I_{2} \sin 2 \varphi$ of $I(\varphi)$ of figure 1 .

tion and the $h_{1 / n, \max }^{(0)}$ corresponding to them determined. For a further calculation, each of the spectral components is changed separately by means of

$$
\begin{gathered}
I_{k}^{(1)}=I_{k}^{(0)}+\Delta I_{k}^{(0)}, \\
\left|\Delta I_{k}^{(0)}\right| \ll I_{k}^{(0)} \quad(k=1,2, \ldots),
\end{gathered}
$$

giving the maximum step-heights $h_{1 / k, \max }^{(1)}$. Because of the linear relationship $h_{1 / k \text {, max }}\left(I_{k}\right)$, the pairs of values $h_{1 / k, \max }^{(0)}, I_{k}^{(0)}$ and $h_{1 / k, \max }^{(1)}, I_{k}^{(1)}$ can be used for a first linear interpolation of $I_{k}^{(2)}(k=1,2, \ldots)$, which approximate the real spectral components $I_{k}$ better than the initial set. This procedure can be repeated. It converges towards the consistent set $I_{k}$ the more quickly the better the linearity of $h_{1 / k, \max }\left(I_{k}\right)$ is satisfied.

In order to prove the practicability of this approximation scheme we have tried to re-construct the $I(\varphi)$-relation, figure 1 , by evaluation of the maximum step-heights $h_{1 / k, \text { max }}(1 \leqslant k \leqslant 5)$, as shown in figure 3 . The result $I_{\text {approx }}(\varphi)$ obtained using a second order interpolation is shown in figure $5 a$. For the purpose of comparison, the real $I(\varphi)$-relation, figure 1 , is plotted in addition. The deviations between $I(\varphi)$ and $I_{\text {approx }}(\varphi)$ correspond mainly to the restriction of the spectrum to the first five components. This result can be seen from figure $5 b$ where $I(\varphi)$-relations are plotted for various integers $l$ of the highest component considered in the expansion of $I(\varphi)$ with respect to $\varphi$. It is this effect which restricts the precision of the interpolation-method. Therefore, the 
approximation becomes better as more heights of subharmonic steps are available. Nevertheless, the results in figure 5 show that this method for an approximate calculation of the current-phase spectrum $I_{k}(k=1,2, \ldots)$, by means of the evaluation of the rf-induced step-structure of given currentvoltage characteristics is practicable.

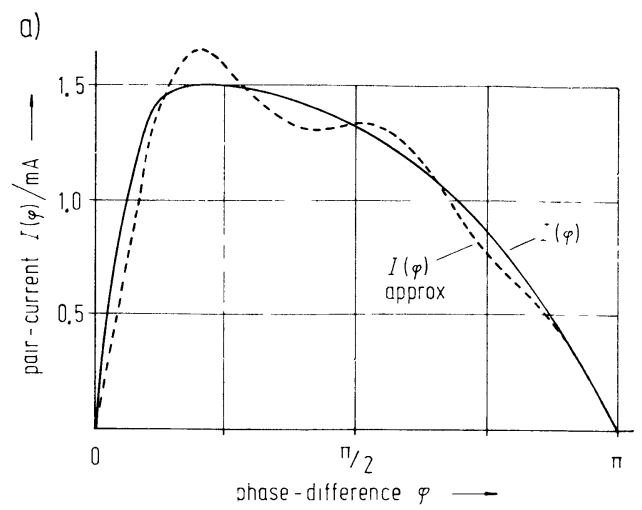

b)

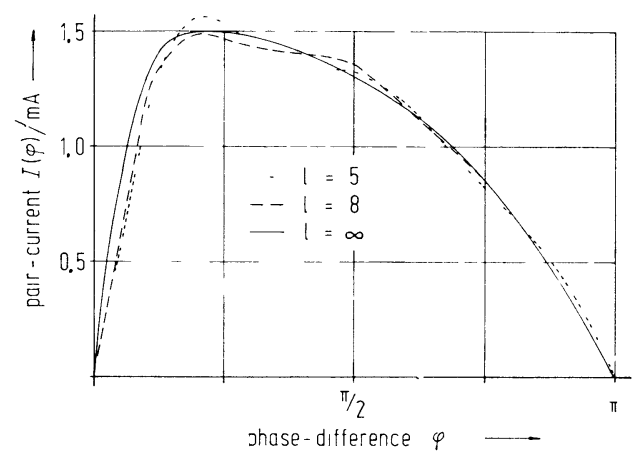

FIG. 5. - a) Comparison of the re-constructed current-phase relation $I_{\text {approx }}(\varphi)$ and the original relation $I(\varphi)$, figure 1 . b) The effect of truncating the series $\sum I_{k} \sin k \varphi$ behind the component $I_{l} \sin l \varphi$ for various integers $l$.

5. Influence of a circuit-capacitance. - It must be emphasized that the step-structures may be modified by means of the influences of reactive circuit elements [2]. Finally we report on some results of calculations based on a current-balance equation which contains an additional capacitive current $C(\hbar / 2 \mathrm{e})$ $\mathrm{d}^{2} \varphi / \mathrm{d} t^{2}$ on the left hand side of eq. (1) where $C$ denotes a circuit-capacitance. These results are compared to those which are based on the use of a sinusoidal current-phase relation $I(\varphi)=I_{1} \sin \varphi$, a situation recently studied by Hamilton and Johnson [2].

Several of our results are shown in figure 6 where $f(\varphi)$ denotes a current-phase relation plotted in figure 1 . The quasiparticle resistance $R_{\mathrm{q}}$ was varied in the range $6.7 \mathrm{~m} \Omega \geqslant R_{\mathrm{q}} \geqslant 2.5 \mathrm{~m} \Omega$. The step-structure of the corresponding current-voltage characteristics is modified continuously in this range, not shown in the figure. The subharmonic steps visible in the characteristics, which are related to the sinusoidal relation $I(\varphi)=I_{1} \sin \varphi$, are caused by the relative large capacitive term. Therefore, the current-voltage characteristics related to $f(\varphi)$ exhibit a mixed step-structure due to both the influences of the capacitive-

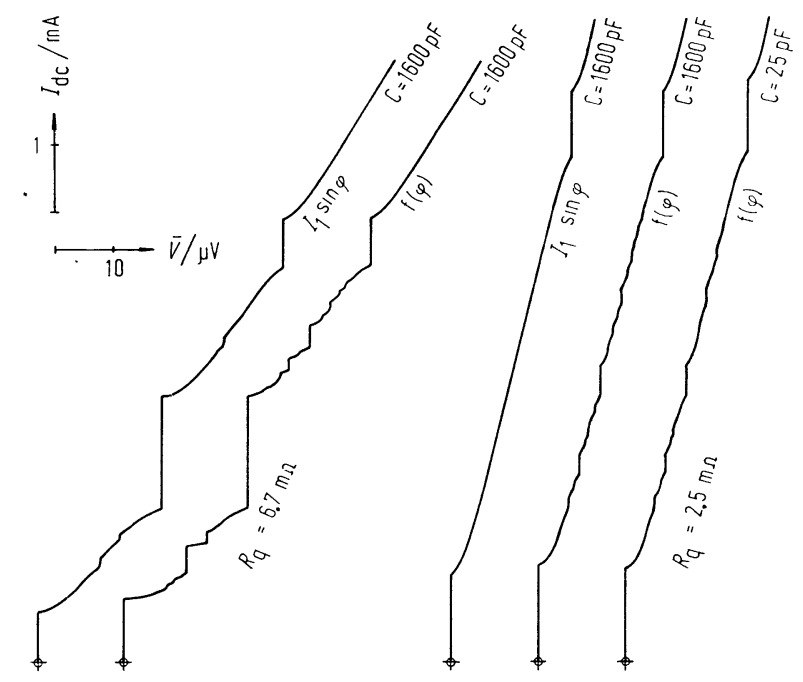

FIG. 6. - Comparison of step-structured current-voltage characteristics due to the non-sinusoidal current-phase relation $f(\varphi)$ identical with that shown in figure 1 and a sinusoidal relation in order to study the influence of an additional capacitive current term.

current term $(C=1600 \mathrm{pF})$ and the non-sinusoidal current-phase relation $f(\varphi)$. As has been mentioned by Hamilton and Johnson, the optimal subharmonic step-structures arise if $C$ and $R_{\mathrm{q}}$ satisfy the condition $\left(C R_{\mathrm{q}}\right)^{-1} \approx 2 \pi f_{\mathrm{rf}}$. This relation is violated for $R_{\mathrm{q}}=2.5 \mathrm{~m} \Omega, C=1600 \mathrm{pF}$. Accordingly, there are no subharmonic structures in the corresponding current-voltage characteristics related to $I(\varphi)=I_{1} \sin \varphi$ and the remaining structures of the curve due to $f(\varphi)$ are caused by the non-sinusoidal current-phase relation only. This result is supported by further variation of $C$ as shown in figure 6 . Therefore, in an experimental situation, the mixing structures can be reduced towards those due to high order components of the current-phase relation only by shunting the microbridge with a resistance smaller than $R_{\mathrm{q}}$.

6. Conclusion. - The analysis of the generation of subharmonic step-structures in the current-voltage characteristics of short microbridges has been shown to be a practicable way to study the shape of the constitutive pair-current-phase relation $I(\varphi)$ corresponding to it. Hopefully, the applicability of the approximation method for the determination of the spectral components $I_{k}(k=1,2, \ldots)$, of $I(\varphi)$, or equally well the phase-coupling conditions of the microbridge, by means of an evaluation of the stepstructure of the given current-voltage characteristic may be confirmed by experiment.

Acknowledgments. - The authors wish to thank Drs. H.-D. Hahlbohm, A. Hoffmann and S. N. Erné for stimulating discussions. 


\section{References}

[1] FACK, H., Kose, V. and Schrader, H.-J., Messtechn. 79 (1971) 31.

Fack, H. and Kose, V., J. Appl. Phys. 42 (1971) 320.

Russer, P., J. Appl. Phys. 43 (1972) 2008.

[2] Kose, V. E. and Sullivan, D. B., J. Appl. Phys. 41 (1970) 169.

Hamilton, C. A. and Johnson Jr., E. G., Phys. Lett. 41A (1972) 393.

[3] Gregers-Hansen, P. E. and Levinsen, M. T., Phys. Rev. Lett. 27 (1971) 847.

[4] Hahlbohm, H.-D., Hoffmann, A., LübBig, H., Luther, H. and Seeck, S., Phys. Stat. Sol. (a) 13 (1972) 607.
[5] Baratoff, A., Blackburn, J. A. and Schwartz, B. B., Phys. Rev. Lett. 25 (1970) 1096; E 1738.

[6] Bardeen, J. and Johnson, J. L. Phys. Rev. B, 5 (1972) 72.

[7] Rieger, T. J., Scalapino, D. J. and Mercereau, J. E., Phys. Rev. B, 6 (1972) 1734.

[8] Auracher, F. and Van Duzer, T., Appl. Phys. Lett. 21 (1972) 515.

[9] Yeong-du Song and Rochlin, G. I., Phys. Rev. Lett. 29 (1972) 416.

[10] Нанцвонм, H.-D. and Luther, H., Elektron. Rechenanlagen 13 (1971) 167.

\section{RESPONSE OF Sn AND In MICROBRIDGES TO APPLIED $10 \mathrm{GHz}, 70 \mathrm{GHz}, 300 \mathrm{GHz}$ AT TEMPERATURES WELL BELOW $T_{c}$ \\ B. KOFOED and K. SAERMARK \\ Physics Laboratory I, The Technical University of Denmark 2800 Lyngby, Denmark}

\section{DYNAMICS OF A JOSEPHSON JUNCTION IN AN ELECTROMAGNETIC ENVIRONMENT}

B. T. ULRICH and D. G. DEMPSEY

University of Texas, Austin, USA 